\title{
Comparison of mixing between single- and bubbly two-phase systems in a Rushton stirred tank
}

\author{
J. S. Moghaddas ${ }^{1}$, J. Revstedt ${ }^{2,3}$, C. Trägårdh ${ }^{2,4}$ \& L. Fuchs ${ }^{2,3}$ \\ ${ }^{I}$ Sahand University of Technology, P.O. Box 51335/1996, Tabriz, Iran \\ ${ }^{2}$ Lund Institute of Technology at Lund University, Sweden \\ ${ }^{3}$ Division of Fluid Mechanics, P.O. Box 118, SE-221 00 Lund, Sweden \\ ${ }^{4}$ Division of Food Engineering, P.O. Box 124, SE-221 00 Lund, Sweden
}

\begin{abstract}
Mixing is a widely practiced operation to effect distribution, intermingling and homogeneity of matter. Stirred tanks with different geometries (such as in main container tanks, impellers, baffles, etc.) are also widely used to mix different materials. The forces applied by impellers develop overall circulation or bulk flow. Superimposed on this flow pattern, there are turbulent eddies and also molecular diffusion of the fluids, which have a direct effect on the mixing phenomenon into the stirred tank reactors. In this work the mixing field in an aerated stirred reactor with two Rushton turbines was experimentally analysed using the particle image velocimetry technique (PIV). The mixing characteristics were measured at three different impeller rotational speeds: 225,300 and $400 \mathrm{rpm}$, for both single- and bubbly two-phase mixing systems. The bubbles will, due to reflection and refraction of the laser light, cause an uneven and unknown distribution of light intensity in the measurement plane. This can be compensated for by introducing a second tracer with a known concentration. Based on this method the mixing of a determining tracer in liquid-phase in a double Rushton turbine stirred tank is studied. Using the PIV measurement technique and the flow structure of the liquid phase, the pumping capacity of those systems were calculated. The aerated-tounaerated relative power was also calculated from the experimental results and compared with the theoretical values. Reasonable agreement between experimental and theoretical results was obtained.
\end{abstract}

Keywords: mixing, bubbly flow, two- phase flow, stirred reactor, Rushton, PIV. 


\section{Introduction}

Stirred tanks are widely used for the mixing of two miscible fluids in the chemical, food and process industries. Our understanding of the flow structure, the behaviour of stirred systems and the basic mixing mechanisms has improved over recent years, but there are still some problems associated with the mixing of fluids in bubbly gas-liquid stirred reactors.

The radial pumping flow rate of an impeller, $Q_{r}$, is defined as the volumetric flow rate leaving the turbine blades, for a chosen radial position, $r$, from the centre of the stirred tank, and can be expressed as:

$$
Q_{r}=2 \pi \cdot r \int_{z_{1}}^{z_{2}} \overline{U_{r}} \mathrm{dz}
$$

where $\bar{U}_{r}$ is the average velocity component in the radial direction. The integration is often performed over the blade height. Assuming $\bar{U}_{r}$ to be proportional to the blade tip speed, $\overline{U_{r}} \propto \pi N D$, then the pumping capacity near the blade can be expressed as in Eq. (2) (Holmes et al. [4]):

$$
Q_{p}=N_{q} N D^{3}
$$

where $N$ is the turbine rotational speed, $N_{q}$ is called the pumping number and $D$ is the impeller diameter. Comparing the results of different studies for singlephase flow in the stirred tanks with Rushton turbines, at the impeller tip where there is no fluid entrainment, the values of the pumping number varied between 0.75 and 1 (Wu and Patterson [7]). The value of $Q_{r}$ can be assumed to be equal to $Q_{p}$ near the blade tip. Therefore, by applying Eqs. (1) and (2) the value of the pumping number can be determined by extrapolating the data for the normalised pumping radial flow rate $\left(Q_{r} / N D^{3}\right)$, as a function of the normalised radius $(r / R)$, in a fully developed flow region of a discharging jet.

The stirring power input for the unaerated single-phase case, $P_{u}$, can be calculated using Eq. (3) (Guillard et al. [3]):

$$
P_{u}=P_{0} \cdot\left(\rho N^{3} D^{5}\right) \cdot\left(\frac{N B_{i m p}}{V_{L i q}}\right)
$$

where $P_{0}$ is the power number and $\rho$ is the density of the continuous phase, $N B_{m p}$ is the number of impellers (two in this study) and $V_{L i q}$ is the total volume of the liquid in the stirred tank. A value of $P_{0}=5.5$, determined by Guillard et al. [3] for the same stirred reactor, was used in the present study.

Trägårdh [6] used an extensive $k-\varepsilon$ numerical model to solve the equation of motion in order to calculate the gas void fraction, concentration, velocity and energy dissipation rate fields in an aerated stirred tank. He showed that the mass 
transfer is higher in the regions of high shear stress, such as near the wall and the interface between the rapidly accelerating "jet" from the turbines and the slow rotating flow. He also stated that the gas void fraction is higher in the region closest to the turbine. In calculating the aerated power, $P_{a}$, he used the following equations:

$$
\begin{array}{ll}
P_{a}=P_{u}\left(1-12.6 N_{G}\right) & \mathrm{N}_{\mathrm{G}}<0.035 \\
P_{a}=P_{u}\left(0.62-1.85 N_{G}\right) & \mathrm{N}_{\mathrm{G}}>0.035
\end{array}
$$

where $N_{G}=Q_{g} / N D^{3}$ is the gas flow number and $Q_{g}$ is the gas flow rate. It can be seen that the reduced aerated power is a function of the gas sparging flow rate, the geometry of the turbine, the rotational speed and the unaerated power, $P_{u}$.

At a constant rotational speed $(N)$ the aerated turbine power $\left(P_{a}\right)$ is lower than the unaerated power $\left(P_{u}\right)$. The subscripts $a$ and $u$ denote the aerated bubbly two-phase and unaerated single-phase flow patterns, respectively. For a low gas void fraction, the ratio between aerated and unaerated pumping flow rates is equal to the ratio between their related powers (Bakker and van den Akker [1]):

$$
\frac{\left(Q_{r}\right)_{a}}{\left(Q_{r}\right)_{u}}=\frac{P_{a}}{P_{u}}
$$

Equation (6) shows that the mechanical agitation, which is a hydrodynamic generator, is affected by air dispersion through the efficiency factor $\frac{P_{a}}{P_{u}}$.

\section{Materials and methods}

The design and the dimensions of the stirred tank with two Rushton impellers of standard geometry, which were used in this work, are shown in Figure 1. The stirred tank consists of a flat-bottomed glass cylinder (refractive index 1.47 at a wavelength of $587 \mathrm{~nm}$ ) an inner diameter $T$ of $0.30 \mathrm{~m}$. Four vertical baffles are symmetrically placed around the tank wall, each with a width of one-tenth of the tank diameter $\left(\ell_{\text {baffle }}=0.1 T\right)$. The Rushton stirrer with two impellers, which is of standard design with a diameter $(D)$ equal to one-third of the tank diameter $(D / T=1 / 3)$, is located with a clearance from the tank bottom of about half of the tank diameter $\left(C_{l}=0.55 T\right)$. The upper impeller is placed $\Delta C=0.7 T$ above the lower one. A pump drives the turbines and the stirring speed is measured using a calibrated digital oscilloscope. In order to reduce optical refractive index effects at the cylindrical surface of the tank, it is placed in a square glass vessel. The stirred tank is filled with tap water as the main continuous phase fluid, the surface of which is $C_{2}=0.55 T$ above the upper impeller. The square vessel is also filled with water to reduce light refraction at the interface. For more details on the geometry of the stirred tank used in this study see Guillard et al. [3]. 


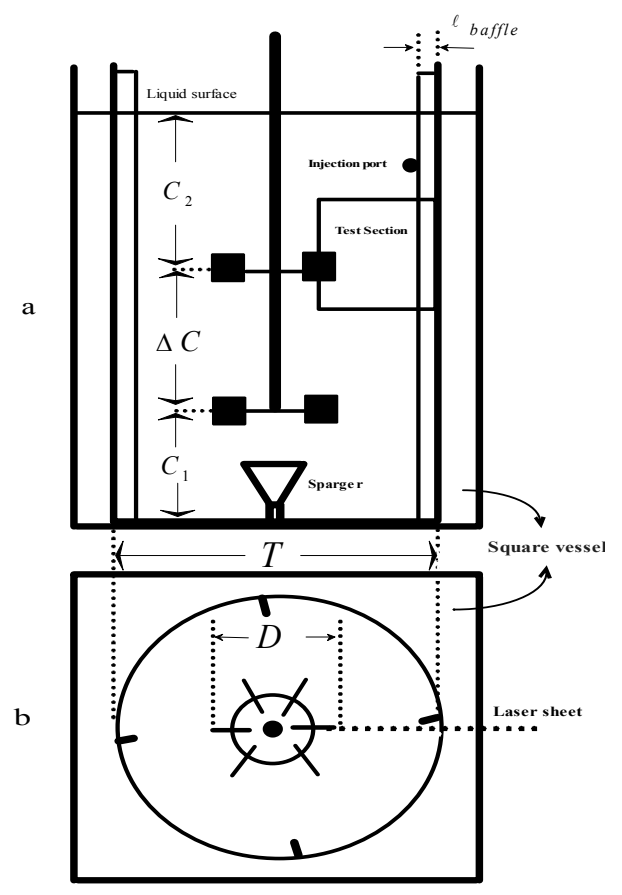

Figure 1: $\quad$ Stirred tank and impellers: a) side view, b) top view.

For the bubbly two-phase flow measurements, air bubbles are introduced, at a superficial gas velocity of $3.96 \times 10^{-4} \mathrm{~m} / \mathrm{s} 50 \mathrm{~mm}$ below the bottom impeller, through a sparger with a diameter of $3 \mathrm{D} / 4$ with 30 active orifices at the chosen gas flow rate.

A double-cavity $2 \times 25 \mathrm{~mJ} \mathrm{Nd:Yag} \mathrm{(Continuum)} \mathrm{pulsed} \mathrm{laser} \mathrm{is} \mathrm{used} \mathrm{to}$ produce a beam at a wavelength of $532 \mathrm{~nm}$. The laser beam passes through a plano-concave lens to produce a two-dimensional vertical sheet of light $(2 \mathrm{~mm}$ thick). Velocity measurements of the liquid phase using PIV were performed and 3 different rotational speeds of the impellers: 225, 300 and $400 \mathrm{rpm}$, were employed. For the determination of the average velocity a CCD camera captured 500 instantaneous images in each case.

In the measurements, the water in the tank was seeded with Rhodamine-B fluorescent tracer particles, $1-20 \mu \mathrm{m}$ in diameter. The flow fields were measured at $23^{\circ}, 30^{\circ}$ and $37.5^{\circ}$ angles behind the impeller blades for the three chosen rotational speeds of the impellers, i.e. 225, 300 and $400 \mathrm{rpm}$, respectively. A CCD camera with two long-pass filters, OG-550 and OG-570 (Melles Griot, Irvine, CA, USA) were used to capture the fluorescent signals from the particles. By masking the scattered signals from the bubbles using the two filters, only the signal from the seeding particles will be captured. The PIV image processing of the liquid continuous phase was performed using multipass interrogation windows decreasing from $32 \times 32$ to $16 \times 16$ pixels. 
The aerated-to-unaerated relative power $\left[\frac{P_{a}}{P_{u}}\right]$ was determined experimentally using Eq. (6), and theoretical values were calculated by applying Eqs. (3) and (4).

\section{Results and discussion}

The flow pattern and the effects of aeration, for an impeller rotational speed of $300 \mathrm{rpm}$, are shown in more details in Figure 2. Figure 2a shows the normalised radial mean velocity profiles at a chosen streamwise location below the impeller as a function of the normalised distance from the blade's centreline, $2 \mathrm{z} / \mathrm{b}=-2.36$ for both single- and bubbly two-phase flow systems. It can be seen that the normalised radial velocities of the continuous liquid phase in the bubbly twophase flow system are lower than those found in single-phase flow. Below the impeller region included higher volumetric gas void fraction according to the visualization, which discussed earlier. A comparison of the axial mean velocity results in a chosen normalised radius close to the wall, $r / R=2.8$ is shown in Figure $2 b$. The bubbles enhance the axial velocity of the liquid continuous phase. Near the wall it could be expected that the influence of the impellers on the motion of the bubbles was less than in the central part of the tank.

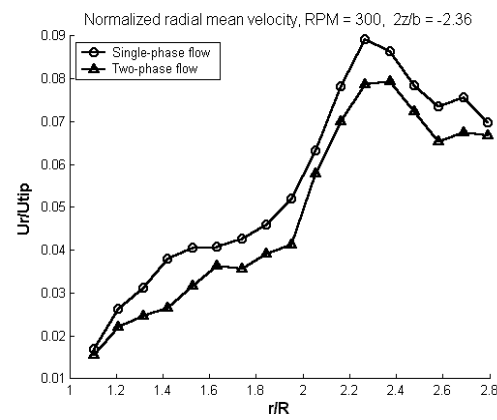

(a)

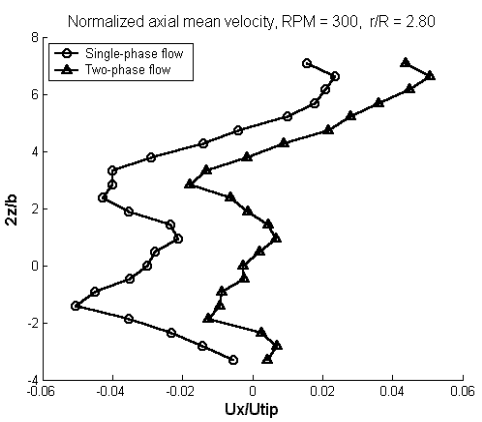

(b)

Figure 2: Comparison of the normalised mean velocity profiles in singleand two-phase flow systems for an impeller speed of $300 \mathrm{rpm}$.

a) Radial profiles, $2 \mathrm{z} / \mathrm{b}=-2.36$, b) axial profiles at $\mathrm{r} / \mathrm{R}=2.80$.

Figure 3 shows the centreline radial mean velocity profiles for the three impeller rotational speeds investigated in both single- and bubbly two-phase flow systems. The velocity data are normalised by the tip velocities, $U_{t i p}=\pi \cdot N \cdot D$, at each impeller speed. The non-random velocity fluctuation caused by the periodic passage of the impeller blades makes the flow structure in the region close to the impeller $(1<\mathrm{r} / \mathrm{R}<1.5)$ more complicated. Stoots and 
Calabrese [5] found that the extent of periodicity in the radial direction is from $\mathrm{r} / \mathrm{R}=0$ to 1.7 and is controlled equally by the radial and tangential components of mean velocity, which shows very good agreement with the presented results in this study. The extent of periodicity in the mean velocity results was found for all three-impeller rotational speeds. However, as the flow field in this range is blade angle dependent, some differences for the mean velocities were noted between different impeller speeds. In this region an accurate measurement of the velocity field is very difficult due to the effect of strong out-of-plane motion due to the swirl. The influence of the bubbles on the centreline mean velocity profile was found to be more pronounced at the highest impeller speed (400 rpm) than at the others.

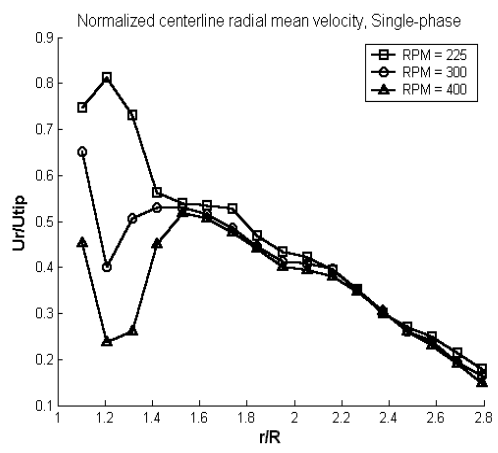

(a)

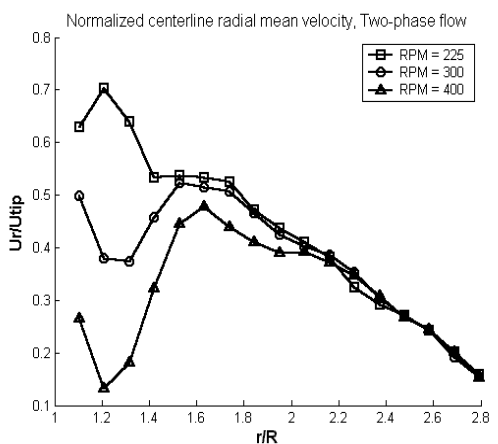

(b)

Figure 3: Centreline radial mean velocity for three different rotational speeds, 225, 300 and $400 \mathrm{rpm}$. a) Single-phase flow, b) two-phase flow.

The normalised pumping flow rate at each speed of rotation for both singleand two-phase flow systems is presented in Figure 4. The results show that the normalised pumping flow rate is almost independent of the impeller speed in the single-phase flow system. The highest impeller speed (400 rpm) shows slightly different values than those for the other speeds. The values of normalised pumping flow rate for the bubbly two-phase flow system show some variation with the impeller speed and the distance from the blade. The effect of aeration on the pumping flow rate is the direct result of changes in the impeller hydrodynamics and the flow pattern.

Table 1 gives the calculated tip pumping numbers for all cases. In the case of single-phase flow it can be observed that the pumping number remains almost constant, with a mean value of 0.89 , independent of the impeller rotational speed. These results are in very good agreement with the values obtained by $\mathrm{Wu}$ and Patterson [7]. The values of pumping number for the case of two-phase flow differ by the factor aerated-to-unaerated power ratio, which is the same effect as that on the normalised radial pumping flow rate. 


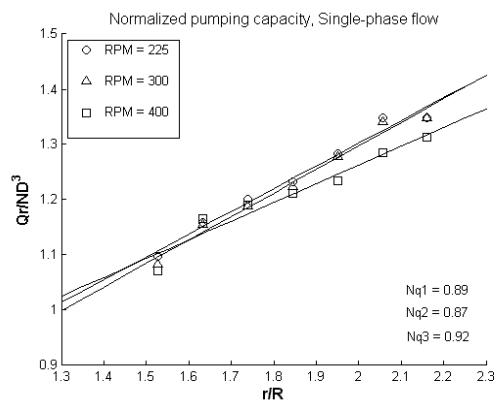

(a)

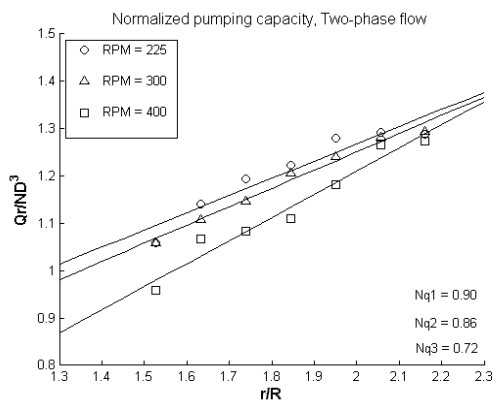

(b)

Figure 4: Normalised pumping flow rate for 3 different rotational speeds, 225, 300 and $400 \mathrm{rpm}$. a) Single-phase flow, b) two-phase flow.

Table 1: Calculated tip pumping numbers for 3 different rotational speeds, 225, 300 and $400 \mathrm{rpm}$.

\begin{tabular}{llll}
\hline RPM & $\mathbf{2 2 5}$ & $\mathbf{3 0 0}$ & $\mathbf{4 0 0}$ \\
\hline Single-phase flow & 0.89 & 0.87 & 0.92 \\
Two-phase flow & 0.90 & 0.86 & 0.72 \\
\hline
\end{tabular}

Table 2: $\quad$ Calculated mixing characteristics of single- and two-phase flow for three different rotational speeds, 225, 300 and $400 \mathrm{rpm}$.

\begin{tabular}{lrrr}
\hline RPM & \multicolumn{1}{c}{ 225 } & \multicolumn{1}{c}{ 300 } & \multicolumn{1}{c}{ 400 } \\
\hline $\mathrm{N}\left(\mathrm{s}^{-1}\right)$ & 3.75 & 5 & 6.67 \\
$\mathrm{U}_{\text {tip }}(\mathrm{m} / \mathrm{s})$ & 1.18 & 1.57 & 2.09 \\
$\mathrm{Re}_{\text {imp }}$ & $3.75 \times 10^{4}$ & $5.00 \times 10^{4}$ & $6.67 \times 10^{4}$ \\
$\mathrm{~N}_{\mathrm{G}}$ & $7.66 \times 10^{-3}$ & $5.67 \times 10^{-3}$ & $4.25 \times 10^{-3}$ \\
$\mathrm{Pu}(\mathrm{W} / \mathrm{kg})$ & 0.12 & 0.30 & 0.72 \\
$\mathrm{~Pa} / \mathrm{Pu}$ & 0.90 & 0.93 & 0.95 \\
$\mathrm{~Pa} / \mathrm{Pu}(\mathrm{Eq} .6)$ & 0.97 & 0.96 & 0.93 \\
\hline
\end{tabular}

Table 1 gives the calculated tip pumping numbers for all cases. In the case of single-phase flow it can be observed that the pumping number remains almost constant, with a mean value of 0.89 , independent of the impeller rotational speed. These results are in very good agreement with the values obtained by $\mathrm{Wu}$ and Patterson [7]. The values of pumping number for the case of two-phase flow differ by the factor aerated-to-unaerated power ratio, which is the same effect as that on the normalised radial pumping flow rate.

At the aeration rate used the aerated stirring power in put $\left(\mathrm{P}_{\mathrm{a}}\right)$ decreases with increasing impeller speed due to the decrease in the gas flow number $\left(N_{G}\right)$, see Table 2. The relative power consumption was shown to be greater than $90 \%$ for all three impeller speeds without a strong drop, as was found by Bakker and van 
den Akker [2], in the range of $0.02<N_{G}<0.04$. Because in this study the values of gas flow numbers for every impeller speeds were less than the critical number of 0.02 found by them. A comparison between the theoretical and average experimental values of $\mathrm{P}_{\mathrm{a}} / \mathrm{P}_{\mathrm{u}}$ shows quiet good agreement, spatially, for the two higher impeller speeds. Table 2 gives the calculated mixing characteristics of single- and bubbly two-phase flow for the three different rotational speeds.

\section{Conclusions}

The mixing field in an aerated stirred reactor with two Rushton turbines was experimentally analysed using the particle image velocimetry technique. The mixing characteristics were measured at three different impeller rotational speeds: 225,300 and $400 \mathrm{rpm}$, for both single- and bubbly two-phase mixing systems. Based on PIV results, the pumping capacity of the systems was calculated. The aerated-to-unaerated relative power was also calculated from the experimental results and compared with the theoretical values. Reasonable agreement between experimental and theoretical results was obtained.

\section{Acknowledgements}

The authors are grateful for the partial financial support from Iranian Ministry of Science, Research and Technology and the Swedish Foundation for Strategic Research, program for Multiphase Flow.

\section{References}

[1] Bakker A; van den Akker HEA (1994a) A computational model for the gasliquid flow in stirred reactors. Trans IChemE 72: 594-606.

[2] Bakker A; van den Akker HEA (1994b) Gas-liquid contacting with axial flow impellers. Trans IChemE 72: 573-582.

[3] Guillard F; Trägårdh C; Fuchs L (1999) A study of turbulent mixing in a turbine-agitated tank using a fluorescence technique. Experiments in Fluids 28: 225-235.

[4] Holmes DB; Voncken RM; Dekker JA (1964) Fluid flow in turbine-stirred, baffled tanks-I Circulation time. Chemical engineering Science 19: 201208.

[5] Stoots CM; Calabrese RV (1995) Mean velocity field relative to a Rushton turbine blade. AIChE Journal 41: 1-11.

[6] Trägårdh Ch. (1988) A hydrodynamic model for the simulation of an aerated agitated fed-batch fermentor. Proceeding of the $2^{\text {nd }}$ International Conference on Bioreactor Fluid Dynamics: 117-134.

[7] Wu H; Patterson GK (1989) Laser-Doppler measurements of turbulent-flow parameters in a stirred mixer. Chemical Engineering Science 44: $2207-$ 2221. 